\title{
La méthode directe en Grèce pour l'enseignement du français : regard particulier sur les niveaux avancés - Le Cours complémentaire de Georges Dimitracopoulos
}

The Direct Method in Greece for the teaching of French: a special look focus on at the advanced levels - tThe complementary Course of Georges Dimitracopoulos

Fryni Kakoyianni-Doa et Monique Monville-Burston

\section{(2) OpenEdition}

Journals

\section{Édition électronique}

URL : https://journals.openedition.org/dhfles/7577

DOI : $10.4000 /$ dhfles. 7577

ISSN : 2221-4038

Éditeur

Société Internationale pour l'Histoire du Français Langue Étrangère ou Seconde

Édition imprimée

Date de publication : 1 décembre 2020

Pagination : 299-316

ISSN : 0992-7654

Référence électronique

Fryni Kakoyianni-Doa et Monique Monville-Burston, « La méthode directe en Grèce pour

l'enseignement du français : regard particulier sur les niveaux avancés - Le Cours complémentaire de Georges Dimitracopoulos », Documents pour l'histoire du français langue étrangère ou seconde [En ligne], 64-65 | 2020, mis en ligne le 05 mars 2021, consulté le 25 mars 2023. URL : http:// journals.openedition.org/dhfles/7577 ; DOI : https://doi.org/10.4000/dhfles.7577

Ce document a été généré automatiquement le 25 mars 2023.

Tous droits réservés 


\section{La méthode directe en Grèce pour l'enseignement du français : regard particulier sur les niveaux avancés - Le Cours complémentaire de Georges Dimitracopoulos}

The Direct Method in Greece for the teaching of French: a special look focus on at the advanced levels - tThe complementary Course of Georges Dimitracopoulos

Fryni Kakoyianni-Doa et Monique Monville-Burston

\section{Introduction}

On sait que le mouvement de la réforme (MR) a été déterminant dans l'enseignement des langues étrangères par l'instauration d'innovations pratiques, la formulation de suggestions théoriques et l'éclosion d'associations et de revues professionnelles. Le MR a eu le soutien de nombre d'enseignants à travers toute l'Europe mais ses principes ont été appliqués à des degrés divers et durant des périodes de longueur variable dans les différents pays où il s'est implanté. Ces questions ont fait l'objet de travaux dans les contextes allemand, français, britannique et hors d'Europe, aux USA (par exemple Puren 2012, Christ 1990, Reinfried 1999, Howatt \& Smith 2014, Mombert 2014). Les études sont plus rares pour d'autres pays. C'est le cas de la Grèce, qui nous intéresse ici. L'étude historique des contenus de l'enseignement du français langue étrangère $y$ concerne en général l'interculturalité et non pas les méthodologies préconisées par les instances ministérielles (Mytaloulis 2016: 606). Nous espérons dans cet article aider à remédier à cette carence. Nous nous proposons d'éclairer ce qu'a été le MR en Grèce de deux points de vue: (1) en analysant dans la première partie du $\mathrm{XX}^{\mathrm{e}}$ siècle les orientations et réglementations didactiques qui s'expriment dans les textes officiels du ministère de l'Éducation; (2) à la suite de l'étude entamée dans Kakoyianni-Doa \& 
Monville-Burston (2019), en posant la question de la nature de la méthodologie directe aux niveaux avancés. s'échelonnent entre 1902 et 1961. Le but est a) de définir ce que recouvre le terme amesi epoptiki didaskalia (désignation en grec de l'enseignement par la méthodologie directe) et b) de déterminer comment ces instructions ont évolué au fil des ans. La seconde partie est une analyse du manuel le plus avancé de la série Le français par la lecture et la conversation $(F L C)$ dont l'auteur, Georges Dimitracopoulos, est un partisan déclaré de la méthodologie directe. La structure de l'ouvrage, ses caractéristiques générales et le format des leçons sont examinés et discutés.

\section{Le mouvement de la réforme}

Le MR a reçu des appellations variées, dont "méthode directe » en France. Pour clarifier la discussion, la terminologie proposée par Puren (2012:77) et soutenue par Howatt et Smith (2014: 84) a été adoptée dans cet article. "Méthodologie directe" (MD) est utilisé pour désigner globalement les différentes démarches employées par les enseignants se réclamant du MR et appelées ici «méthodes». On parlera donc de méthodes naturelle, directe, orale, interrogative, répétitive, intuitive, etc. En Grèce, la MD a été dénommée par les instances officielles amesi epoptiki didaskalia ('enseignement direct par les sens'). L'adjectif amesos signifie 'direct' et l'adjectif epoptikos, selon Babiniotis, se réfère à tout ce qui sert la perception par des impulsions directes, à tout ce qui sert l'esprit en fournissant des stimuli immédiats et en permettant une observation immédiate (2002: 664).

Pour la présente étude, la date initiale de 1902 a été retenue car c'est à cette date qu'apparaissent pour la première fois des instructions officielles sur la pratique d'exercices de prononciation et de lecture de mots/phrases pour le cours de français (méthode orale) (Journal Officiel [dorénavant JO], $\mathrm{n}^{\circ}$ 154, 22-08-1902). La date finale de 1961 a été choisie parce que des manuels appliquant la MD ont été publiés et réédités jusqu'à cette date (cf. Mytaloulis $2016: 614$ ).

5 Pour effectuer la présente recherche, la banque de données en ligne de la «Collection historique d'ouvrages scolaires grecs » a été utilisée ${ }^{1}$. Tous les décrets qui concernaient l'enseignement du français ont été soigneusement inspectés année par année et leur contenu classifié. Cela a permis de montrer, d'une part, les principales caractéristiques de la MD en Grèce, et d'autre part, de dresser un tableau des types d'activités recommandés pour chaque niveau dans les écoles secondaires.

\section{Histoire des réglementations didactiques : décrets et instructions}

\section{Éveil de l'intérêt pour l'enseignement de la langue parlée (1831-1902)}

6 L'importance de l'enseignement de la langue parlée, qui deviendra une caractéristique fondamentale de la MD, a été reconnue par certains pédagogues des $\mathrm{XVIII}^{\mathrm{e}}$ et $\mathrm{XIX}^{\mathrm{e}}$ siècles (Puren 2012, Reinfried 1999, Besse 2012), et en Grèce l'attention à l'oralité est 
aussi à noter chez Anastasios Herculides, l'auteur du premier manuel de français (Isigitis tis gallikis glossis : 'Introduction à la langue française', 1831) (Caravolas $2015: 3$ ). Mais en Grèce comme ailleurs en Europe, il faudra attendre la fin du XIX siècle pour que s'établisse la MD, avec ses démarches variées, dont la primauté de la langue parlée. L'officialisation de la MD commence dans différents pays européens entre 1897 et 1911 (Berré \& Besse 2012 : 72). En Grèce, elle viendra en 1907 mais déjà certains pédagogues militent pour l'adoption de nouvelles pratiques dans l'enseignement, en particulier pour un enseignement basé sur l'oral, conseillé par les directives du ministère concernant la formation des maitres. En 1902, par exemple, on lit dans le JO que dans les trois classes des écoles qui forment les futures institutrices, il faut proposer « des exercices pour enseigner à parler aussi bien qu'à écrire » dans les cours de français (JO, $\mathrm{n}^{\circ} 154,22-08-1902$, p. 42-43).

\section{Premiers jalons pour l'introduction de la MD (1905-1913)}

7 Ce qui constitue la grande nouveauté dans les années qui suivent, c'est l'introduction de l'image pour encourager l'oralité et l'apprentissage d'un vocabulaire usuel. Par exemple, l'École de commerce de Pirée qui souhaite dispenser un enseignement 'pratique', préconise pour la conversation l'utilisation des tableaux de l'artiste viennois Adolf Hölzel (originellement destinés à l'enseignement de l'allemand L1), dans son programme de 1905-1906. Dans le décret du ministère de 1907 apparaît l'expression epoptikin kai praktikin didaskalian, "enseignement par les sens et enseignement pratique ", qu'on recommande pour les cours de français. Ce décret institue aussi un système national qui impose pour la première fois un manuel par matière/classe dans les écoles helléniques et les gymnases ${ }^{2}$. Le français était enseigné à partir de la $2^{\mathrm{e}}$ année de l'école hellénique. On prescrit trois ouvrages différents, dont l'État a le monopole ${ }^{3}$. Le premier, appelé 'principal' (stichiodes) pour les $2^{\mathrm{e}}$ et $3^{\mathrm{e}}$ années de l'école hellénique était partiellement conçu dans l'esprit de la MD. Il devait présenter des descriptions brèves et des récits concernant la vie quotidienne de l'élève et des gravures urbaines de Hölzel. Chaque leçon devait comprendre un questionnaire (méthode orale/ interrogative) et des exercices. Ceux-ci étaient accompagnés d'explications grammaticales brèves rédigées en grec. On dérogeait donc là au principe unilingue de la MD. Le deuxième ouvrage, appelé 'méthode' (methodos) était destiné au gymnase. Il devait contenir lui aussi des images de Hölzel, ainsi que des devoirs à faire à la maison, et proposer par ailleurs, en bas de page, des remarques concernant la grammaire. Le troisième ouvrage était une grammaire entièrement rédigée en grec qui donnait les règles « absolument nécessaires » (JO, n 139A/12-07-1907). Au final, on voit que l'image gagne sa place comme support de la L2 orale dans les programmes, mais que la MD n'y est pas encore explicitement citée comme le modèle d'enseignement. Dans les classes supérieures pour lesquelles les contenus des enseignements ne sont pas mentionnés, si ce n'est une grammaire simplifiée, la méthode traditionnelle (MT) restait en vigueur ( $c f$. Provata \& Vihou $2018: 326)$.

\section{Établissement de la MD (1914-1929)}

8 En 1914, le ministère de l'Éducation refond le contenu des diverses matières d'enseignement. C'est dans le texte du décret de cette année-là qu'apparaît l'expression amesi epoptiki didaskalia ('enseignement direct par les sens') pour l'enseignement du 
français, aux deux premiers niveaux (JO, $\mathrm{n}^{\circ}$ 369, 10-12-1914, p. 2003-2004). Les instances ministérielles veulent promouvoir un enseignement fondé sur l'action naturelle qui accorde la priorité à l'oral, à l'utilisation directe de la L2, à la prononciation, et (toujours) à la conversation basée sur des tableaux muraux. Au programme figurent donc des exercices de phonétique, de lecture avec interprétation par interaction entre professeur et élève par questions-réponses, des dictées, et des récitations de poèmes (JO, n 343A, 24-11-1914).

9 Concernant les manuels, les instructions officielles de 1923 préconisent des ouvrages publiés à l'étranger: pour le niveau débutant Dent's First [and second] French Book, de Sines Alge et Walter Ripmann ; pour les classes de $3^{\mathrm{e}}$ et $4^{\mathrm{e}}$ années, des livres de français L1, Francinet - Livre de lecture courante, et Choix de lectures - Cours moyen $1^{\text {er }}$ degré de Mironneau, mais également pour les cours plus avancés des extraits d'œuvres littéraires classiques. (JO, $\mathrm{n}^{\circ}$ 6, 21-01-1923) .

\section{Structuration des programmes - Consolidation de la MD aux niveaux élémentaires et moyens (1929-1935)}

Le décret de 1929 restructure le système éducatif grec dans son ensemble et, pour l'enseignement du français, représente une étape-clé dont les aspects curriculaires seront repris dans le programme analytique de 1931. Un cycle de six ans, appelé 'gymnase', est créé pour les études secondaires (JO, n 309/A, 24-8-1929). On lance un nouveau concours pour la rédaction d'un manuel de français intégral pour chaque niveau/année. Les trois ouvrages prescrits en 1907 (cf. supra) sont donc supprimés pour des raisons d'économie, dit-on, et de rigueur et de progression logique dans l'enseignement. Le ministère est convaincu que les élèves n'atteignent pas les objectifs voulus et demande que soient appliquées à la lettre toutes les directives qui avaient été négligées auparavant, ce qui laisse à penser que les principes de la MD n'étaient pas entièrement suivis. Des instructions précisent le découpage des manuels et la répartition des chapitres. Par exemple pour les $1^{\text {ère }}$ et $2^{\mathrm{e}}$ années, l'ouvrage doit contenir 32 leçons dont seize enseignées par la méthode directe, et seize (consacrées à la lecture) par la méthode indirecte. Chaque cours doit comporter deux étapes : 1) à partir d'une image support (tableau de Hölzel, etc.), échange questions-réponses entre maitre et élève ; 2) lectures diverses. La grammaire ne doit jamais faire l'objet d'un cours entier. Il est recommandé de porter une attention spéciale à la prononciation des sons du français problématiques pour les Grecs. Ou encore, pour la $6^{e}$ (et dernière) année, l'ouvrage doit être découpé en 38 leçons dont 17 dédiées a) à l'étude d'une anthologie de morceaux choisis d'auteurs classiques ou de prose philosophique, avec interprétation des textes par le biais de discussions déclenchées par des questions, et b) à une initiation à l'histoire de la littérature. Seize leçons doivent être des cours de conversation et cinq des leçons de synthèse, à savoir des cours d'expression écrite avec analyse des erreurs. On notera que le programme analytique demande aussi que soient enseignés des éléments de civilisation française à travers les textes de lecture proposés. Ce programme établit donc pour le niveau avancé une méthodologie 'mixte' avec sensiblement plus d'écrit que d'oral.

11 Pendant les six années qui suivent, on n'observe pas de véritable changement dans les programmes (JO, n 537/09-11-1935). 


\section{Généralisation de la MD (1937-1961)}

12 Malgré les divers débats méthodologiques qui se déroulent à cette époque et le scepticisme de certains pédagogues vis-à-vis de la MD dans d'autres pays européens, celle-ci ne semble pas être remise en question en Grèce ${ }^{5}$. Ainsi quand l'anglais devient obligatoire dans les gymnases, après la seconde guerre mondiale, le décret de 1953 conseille fortement l'emploi de l'amesos methodos, à savoir la MD. Pour le français les instructions de 1937 recommandent avec encore plus de vigueur que les précédentes la pratique de la phonétique, du rythme et de l'intonation. En 1939 l'amesi epoptiki didaskalia est étendue jusqu'en $5^{\mathrm{e}}$ année, un niveau avancé, et continue à être préconisée en 1941 (JO, nº 197/17-06-1941, p. 985).

Jusqu'en 1960, l'enseignement du français ne fait plus l'objet d'aucun décret particulier. Toutefois dans les décrets de 1961, pour toutes les années du gymnase, on sent que les acquis de la MD sont définitivement adoptés. La MD est citée à nouveau comme modèle à suivre. On insiste que l'élève comprenne, lise et utilise convenablement la langue française à l'oral aussi bien qu'à l'écrit. Pour les premiers niveaux, l'accent est mis sur l'acquisition de la prononciation (phonèmes et rythme). L'utilisation de procédés et de techniques permettant prioritairement l'utilisation de la langue orale sans passer par l'intermédiaire de sa forme écrite est toujours en faveur: L2 en classe, dialogues, descriptions orales d'images, récitation et dictée, ainsi que la mémorisation d'un vocabulaire fondamental qui ne dépasse pas 500 à 600 mots. Ce souci de limitation lexicale répondait sans doute aux critiques adressées aux praticiens de la MD qui présentaient à leurs élèves un vocabulaire exhaustif, mais contribuaient à les décourager 6 .

Aux niveaux moyens et avancés les décrets donnent de semblables conseils. On recommande aussi aux professeurs de ne pas compliquer leur enseignement de la grammaire et de le baser sur l'enseignement oral. Pour la littérature, elle doit être abordée parallèlement au travail oral. Les textes doivent avoir une fonction non seulement pratique, mais aussi formative (connaissance de la vie quotidienne et des mœurs du peuple français, de sa culture et de son histoire) (JO, $\mathrm{n}^{\circ}$ 160/13-09-1961, $\mathrm{p}$. 1503 ; JO, n ${ }^{\circ}$ 55/18-03-1961, p. 555 et JO, n 151/12-09-1961, p. 1443).

L'examen des décrets officiels qui se sont succédé de 1901 à 1961 a montré comment la MD s'est progressivement et fermement implantée en Grèce pour le français, des petits niveaux aux niveaux moyens dans les écoles secondaires. On a pu remarquer que ces décrets étaient souvent moins éloquents sur la forme que devait prendre l'enseignement aux niveaux supérieurs, laissant supposer que la MT y était toujours bien ancrée. Cependant la MD n'y a pas été sans influence. Elle y a pris une forme plus tempérée, celle d'une méthodologie mixte MT-MD. Des compromis étaient nécessaires si on voulait que les langues étrangères contribuent à prodiguer un enseignement humaniste, et non pas seulement pratique.

16 Jusqu'aux années 1960 des pédagogues enthousiastes, dogmatiques même, ont tâché de soutenir la MD et d'en assurer le succès à tous les niveaux. C'est le cas de Georges Dimitracopoulos qui s'est proposé la gageure de concevoir un cours avancé solidement fondé sur la $\mathrm{MD}^{7}$. 


\section{Le Cours complémentaire de Georges Dimitracopoulos}

Le Cours complémentaire (CC) est le manuel le plus avancé de la série FLC dont l'auteur, Georges Dimitracopoulos, est un défenseur zélé de la MD, comme il l'affirme dans les préfaces de tous les manuels de cette série. Il s'adresse aux élèves de l'année terminale du secondaire. Il n'est pas daté mais on peut en situer la parution entre les années 1935 et 1959. Le CC étant le dernier cours de la série, il est probablement le plus tardif ${ }^{8}$. On sait peu de l'auteur si ce n'est qu'il était professeur de français et directeur d'une maison d'édition.

\section{Les préfaces du Cours supérieur et du CC}

Dans la préface de son Cours supérieur (CS) - qui précède le $C C$ mais dont les propos y sont applicables - Dimitracopoulos définit ce qui est central à son approche pédagogique. Sa préface est un manifeste en faveur de la langue familière actuelle et de la langue de la conversation ${ }^{9}$ :

[...] l'élève étudie toute une série d'ouvrages qui le préparent généralement à affronter les difficultés de la langue écrite et à comprendre plus ou moins bien le français littéraire, qui, cependant, remarquons-le en passant, n’a jamais été la langue que de très peu de gens et n'est aujourd'hui la langue de personne. [...] l'élève finit, certes, par lire couramment Corneille et La Fontaine, mais il ne se sent pas capable du tout de lire les annonces de la quatrième page d'un journal ou de converser avec un Français lorsque ce dernier se sert du langage actuel de ses compatriotes et qui est la forme vivante de la langue. (Préface du CS : 9).

Le fait que la langue parlée soit première et ait la fonction sociale d'un instrument de communication doit donc se refléter dans une méthodologie de l'enseignement basée sur l'oral qui ajoute à l'objectif traditionnel un objectif pratique : «[...] on ne doit pas sacrifier l'utile [...] à ce qui ne présente pas une utilité immédiate", écrit Dimitracopoulos (10), ce qui n'implique pas que la langue écrite, et les textes littéraires, doivent être négligés, en particulier aux niveaux avancés. Il est donc important que l'élève comprenne les différences de registre. D'où l'alternance dans le $C C$ entre les lectures (littéraires et autres) et les parties dialoguées, les deux espèces de textes "se complétant l'une l'autre ». L'auteur considère que cette façon de concevoir ses Cours est une "innovation", sans doute en comparaison avec les autres manuels avancés qui sont sur le marché et restent entièrement conformes à la MT. En outre cette alternance aura l'effet bénéfique d'éviter aux élèves le découragement et l'abandon de leurs études de français. On notera que Dimitracopoulos insiste toujours sur l'importance des émotions positives et des facteurs qui encouragent l'élève à étudier ${ }^{10}$. Il souligne en outre (Préface du CC:9) que le but de son livre est d'étendre le vocabulaire de l'élève, de compléter ses connaissances grammaticales et de lui enseigner de nouveaux éléments de civilisation française. Dimitracopoulos ajoute enfin que son ouvrage est " conçu d'après la même méthode et suivant le même esprit que les cours précédents ". Il faut entendre par là qu'il continue à appliquer les principes de la MD. En particulier la L1 de l'élève n'est jamais utilisée, même pas dans les consignes ou les explications en note de bas de page. 


\section{Structure de l'ouvrage et format des leçons}

On observe dans toute la série des cours de Dimitracopoulos une uniformité du format des leçons, du niveau préparatoire aux niveaux avancés (Tableau 1).

Tableau 1 : Plan prototypique d'une leçon (applicable au CC)

\begin{tabular}{|l|l|}
\hline Éléments de la leçon & Fonction de chaque élément \\
\hline Texte & Support de la leçon \\
\hline Questionnaire & $\begin{array}{l}\text { Activités de classe : questions- } \\
\text { réponses entre maître et élèves }\end{array}$ \\
\hline Exercices & Devoirs à faire à la maison \\
\hline $\begin{array}{l}\text { Éléments supplémentaires, selon le type de texte support } \\
\text { (dictée, récitation, anecdote, image) }\end{array}$ & $\begin{array}{l}\text { Fonctions variées selon le type } \\
\text { d'élément supplémentaire }\end{array}$ \\
\hline
\end{tabular}

21 Les diverses démarches de la MD sont engagées dans ces leçons : méthodes directe, orale, interrogative, répétitive, intuitive (par l'aspect). Il va de soi que l'application de la MD intégrale est moins aisée aux niveaux avancés et que des accommodations s'imposent. En particulier le texte support, qui organise autour de lui les activités d'apprentissage, ne peut plus être le point d'arrivée de l'enseignement, mais en devient le point de départ (Puren 2012 : 112). En effet contrairement à ce qui se passe aux niveaux élémentaires la leçon ne peut plus se faire à livre fermé par questions-réponses avant d'aboutir à la lecture. On part plutôt des textes, dont on fait une 'lecture expliquée' et qui servent à l'acquisition de toutes les compétences, de la phonétique à la littérature et à la culture.

\section{Les textes supports}

Les textes supports se répartissent comme suit dans le CC (Tableau 2) :

Tableau 2 : Contenu des textes supports

\begin{tabular}{|l|l|l|}
\hline Type de texte & Nombre & Remarques \\
\hline $\begin{array}{l}\text { Morceaux choisis } \\
\text { littéraires }\end{array}$ & 17 & Auteurs des $17^{\mathrm{e}}, 18^{\mathrm{e}}$ et $19^{\mathrm{e}}$ siècles \\
\hline $\begin{array}{l}\text { Conversations } \\
\text { causeries }\end{array}$ & 27 & $\begin{array}{l}\text { Thèmes : chez le tailleur, le cordonnier, le docteur, le coiffeur, } \\
\text { etc. }\end{array}$ \\
\hline $\begin{array}{l}\text { Exposés de grammaire } \\
\text { Exposés sur la vie } \\
\text { quotidienne }\end{array}$ & 28 & $\begin{array}{l}\text { Ordre des mots, constructions verbales, temps et modes, etc. } \\
\text { géographie de la France, monuments de Paris, etc. }\end{array}$ \\
\hline
\end{tabular}



choisis ", et " courts ». Ils sont effectivement très brefs (une page, parfois deux), et demandent évidemment à être sélectionnés avec discernement si l'on veut que quelques paragraphes forment un tout cohérent. Leur gradation demande aussi beaucoup d'attention. Les extraits du théâtre classique ne viennent que très tard dans le manuel. Tout cela obéit aux principes de la MD : aller du simple au complexe pour ne pas rebuter l'élève : « [...] il n'est pas sans inconvénient, croyons-nous, à forcer l'élève à pâlir pendant un mois sur un même auteur [...]» (ibid. 9). On peut voir dans le CC que l'extrait littéraire est considéré par Dimitracopoulos comme un 'document authentique', utilisé pour développer les compétences de compréhension écrite et encourager l'expression orale, mais a aussi pour but de faire découvrir, même modestement, la culture littéraire et d'être un instrument d'introduction à l'étude des humanités : "[...] il s'agit de savoir bien ce qu'il y a d'exquis dans la littérature française » (ibid. 8). D'où les paragraphes d'une dizaine de lignes à la suite des extraits, qui résument la biographie de l'auteur de l'extrait et indiquent dans quel(s) genre(s) il se spécialise.

\section{Les exposés de grammaire}

Ici, c'est une leçon de grammaire qui sert de texte support. Ces leçons s'adressent à des élèves qui ont déjà une très solide connaissance du français. Elles sont peu nombreuses et courtes (sur deux pages). Elles traitent de points spécifiques que Dimitracopoulos a sans doute jugés difficiles pour les jeunes Grecs : place de l'adjectif, emploi des temps et modes, concordance des temps, constructions verbales. Contrairement aux manuels du même auteur destinés aux niveaux inférieurs, ces leçons n'encouragent pas l'apprentissage inductif. La procédure de présentation est explicite et analytique.

\section{Les textes conversationnels : dialogues et causeries}

Les conversations proposées aux élèves sont des échanges de propos entre deux ou plusieurs personnes, entre amis ou connaissances, ou entre eux et des fournisseurs, des fonctionnaires, etc. Contrairement aux textes littéraires, elles sont longues, entre une page et demie et trois pages. Nous n'avons aucune indication dans la préface sur la façon dont ces conversations étaient exploitées: lues d'abord dans leur entier ou réplique par réplique par le professeur et expliquées si nécessaire, jouées par des élèves? Nous avons cependant une idée des questions de compréhension qui pouvaient être posées si l'on se fie au questionnaire qui suit le texte ( $c f$. infra). Entre amis, le ton de la conversation est familier, enjoué, mais ne va pas jusqu'au tutoiement. Même dans les conversations courantes, la langue n'est jamais dénuée d'apprêt.

La différence entre les textes de conversation et les textes de causerie est que la causerie est une discussion sur un sujet unique (musique, sports, théâtre, etc.). 
Conversations et causeries, quoiqu'artificiellement élaborées, ont pour but de mettre la langue en situation, en traitant de questions pratiques. La nature du langage employé est en conformité avec des types d'activités de la vie courante ou de «formes de vie "; le vocabulaire, les structures et la pragmatique sont dictés par l'usage ${ }^{11}$. Ces textes permettent l'apprentissage d'expressions figées, de collocations, des «formes de parole» que Wilhelm Viëtor considérait comme les éléments fondamentaux du langage, plus essentiels à acquérir que la grammaire.

\section{Les exposés}

Eux aussi sont fabriqués, et comme les causeries, ils se rapportent à un centre d'intérêt unique (toilette, habillement, monument historique, tableaux de la vie à l'étranger). Ils introduisent $\mathrm{du}$ vocabulaire thématique nouveau souvent en liaison avec un texte conversationnel. On est frappé par la quantité d'items lexicaux qu'on demande à l'élève d'assimiler : Puren parle à juste titre d'une «inflation effrénée » du vocabulaire dans les manuels de MD (par exemple, dans le texte sur la musique, on a l'inventaire de tous les instruments). La MD avait en effet tendance à insister sur la connaissance d'un vocabulaire étendu pour éviter que l'élève n'ait recours à la L1 du dictionnaire bilingue. L'accumulation du vocabulaire technique est aussi liée à la préoccupation d'intégrer au maximum l'apprentissage de la L2 au monde concret, familier à l'élève.

\section{L'enseignement de la civilisation}

Cet enseignement se fait a) à travers certains textes (conversationnels et autres), b) par l'intermédiaire de gravures (occupant une page entière) dans les sections du livre intitulées " Documents de civilisation française » (treize en tout) et 3) par des gravures plus petites incorporées aux textes. Les gravures représentent des monuments, musées, rues, et autres lieux urbains célèbres en France, mais aussi des lieux ou événements plus génériques que l'on prend soin de situer en France en le précisant dans la légende, par exemple, "Défilé de mannequins dans une maison de haute couture parisienne ». Les images parisiennes dominent ( $2 / 3 \mathrm{du}$ total). Paris est, en abrégé, la représentation symbolique de la France. De ce point de vue, l'auteur ne fait que se conformer aux directives ministérielles ( $c f$. Provata \& Vihou, $2018: 327$ ).

\section{Questionnaires, exercices, dictées et récitations}

Chaque texte (non-grammatical) est suivi d'un questionnaire. Ces questionnaires sont des déclencheurs de prise de parole par l'élève, mais aussi un moyen de s'assurer que le texte support a été véritablement compris. Les questions sont factuelles et progressent selon la chronologie du texte. Elles ne touchent que le contenu. Ce sont les exercices qui font travailler les formes linguistiques.

31 Les exercices sont des devoirs à faire à la maison. Dans les leçons consacrées à la grammaire, ils sont centrés sur une question ou difficulté unique (cf. Vigner $2017: 10$ ). Dans toutes les autres leçons, ils sont "panachés", hétéroclites: ils rassemblent plusieurs difficultés de nature différente, étroitement liées à des aspects linguistiques $\mathrm{du}$ texte support ( $c f$. Kakoyianni-Doa \& Monville-Burston 2019). Les consignes 
demandent à l'élève de s'interroger sur la phonétique/graphie, la morphologie et la syntaxe, le lexique, la nature et la fonction des mots ou des propositions ${ }^{12}$. On note que l'expression écrite (composition libre sur un sujet lié à la leçon) n'apparaît qu'à partir de la leçon 33 et peu fréquemment. L'expression orale reste centrale, même au niveau supérieur.

Les autres activités, en fin de leçon, sont la lecture-dictée (en prose) et la lecturerécitation (en vers). Elles témoignent de l'importance donnée à la vérification de l'orthographe, à l'apprentissage par cœur d'un beau texte et à une bonne prononciation et une bonne diction, ces compétences étant toutes liées à la méthode orale.

\section{Conclusion}

La présente étude a montré, à travers l'examen des textes officiels du ministère de l'Éducation, le développement de l'emploi de la MD en Grèce entre 1902 et 1961 pour l'enseignement $\mathrm{du}$ français dans les écoles secondaires. Adoptée initialement aux niveaux débutants, cette méthodologie a gagné progressivement les niveaux moyens, sous forme intégrale ou modérée. À la fin de la période considérée, au moment où il semble qu'on ait officiellement cessé de l'utiliser en France, elle a aussi été intégrée aux niveaux avancés en association avec des formes d'enseignement plus traditionnelles qui assuraient une culture humaniste.

L'analyse du CC de Dimitracopoulos a permis d'éclairer comment la méthodologie officielle a pu être mise en pratique après la seconde guerre mondiale dans un cours avancé. On trouve appliqués dans le $C C$ tous les grands principes de la MD. L'apprentissage est direct : la L1 de l'élève n'est jamais utilisée. Corollairement, pour éviter les recours au dictionnaire, le lexique occupe une place essentielle dans l'acquisition. L'usage de la langue parlée est prioritaire : les conversations et causeries sont plus nombreuses que les morceaux littéraires. Les dictées et récitations assurent la correction phonétique et la connaissance de la relation phonie-graphie. L'image tient une place importante: c'est par elle, entre autres, que se fait l'enseignement de la culture. Par son exploitation, orale, on sollicite l'activation de l'intuition des apprenants. La formation humaniste/culturelle de l'élève sans être négligée n'est pas centrale : les contenus pratiques l'emportent sur les contenus littéraires. Enfin, pour que l'élève prenne activement part à son apprentissage, on se soucie de sa motivation. Cependant le traitement du texte littéraire et l'acquisition de la grammaire restent toujours marqués par la MT. Au final, Dimitracopoulos a "innové », comme il le prétendait dans sa Préface, en appliquant les principes de la MD aussi systématiquement que possible au niveau le plus avancé des études françaises.

\section{BIBLIOGRAPHIE}

Sources primaires 
DIMITRACOPOULOS, Georges D. (?). Le français par la lecture et la conversation - Cours supérieur.

Athènes : Éditions Georges.

DIMITRACOPOULOS, Georges D. (?). Le français par la lecture et la conversation - Cours complémentaire.

Athènes : Éditions Georges.

\section{Journal officiel}

$\mathrm{n}^{\mathrm{o}}$ 154, 22-08-1902, Loi relative au programme scolaire.

$n^{\circ}$ 139/A, 12-7-1907, Programme [...] des livres scolaires.

nº 343/A, 24-11-1914, Sur le programme des matières de l'école hellénique et du gymnase.

n $369,10-12-1914$, Sur le programme des matières de l'école hellénique et du gymnase.

$n^{\circ}$ 6, 21-01-1923, Sur le programme des lycées publics et privés.

$\mathrm{n}^{\circ}$ 309/A, 24-8-1929, Sur l'enseignement primaire.

$n^{\circ}$ 537/09-11-1935, Sur le programme de l'enseignement secondaire.

$n^{\circ}$ 197/17-06-1941, Sur le programme et l'emploi du temps des gymnases de six classes, d'ancien type pour l'année scolaire 1940-1941.

$\mathrm{n}^{\circ}$ 160/13-09-1961, Sur le programme et l'emploi du temps du premier niveau des gymnases.

$\mathrm{n}^{\circ}$ 55/18-03-1961, Sur le programme et l'emploi du temps de la $4^{\mathrm{e}}$ année à orientation pratique.

n $151 / 12-09-1961$, Sur le programme et l'emploi du temps de la 5e année à orientation pratique.

\section{Sources secondaires}

BABINIOTIS, Georgios (2002). Dictionnaire du grec moderne. Athènes : Centre de lexicologie EPE.

BERRÉ, Michel \& BESSE, Henri (2012). « Méthodes, techniques d'enseignement du français comme L2 : éléments pour une réflexion historiographique ». Le français dans le monde, 52, 62-77.

BESSE, Henri (2012). « Éléments pour une 'archéologie' de la méthode directe ». Documents pour l'histoire du français langue étrangère ou seconde, 49, 11-30.

BOUQUET, Simon (1999). « De la Méthode Directe aux Investigations philosophiques de Wittgenstein. Savoirs et transferts de savoirs ». Langage et société, 87, nº 1, 41-77.

CARAVOLAS, Jean Antoine (2015). « Le premier manuel de français publié en Grèce ». Documents pour l'histoire du français langue étrangère ou seconde, 54, 63-78.

CHRIST, Herbert (1990). « Pour et contre la méthode directe : Les débats au sein de la l'Association Allemande des Professeurs de Langues Vivantes entre 1886 et 1914 ». Études de Linguistique Appliquée, 90, 9-22.

HowATT, Anthony P. R. \& SMITH Richard (2014). « The History of Teaching English as a Foreign Language, from a British and European Perspective ». Language \& History, 57, 75-95.

KAKOYIANNI-DOA, Fryni \& MONVILLE-BURSTON, Monique (2019). « L'exercice dans une série de manuels FLE grecs des années 1940-1950 : La méthode Dimitracopoulos ». Documents pour l'histoire du français langue étrangère ou seconde, 62-63, 449-471.

MOMBERT, Monique (2014). « Les revues de langues vivantes en France et en Allemagne autour de 1900 : la fabrique d'une opinion publique enseignante ». Schweizerische Zeitschrift für Bildungswissenschaften, 36, 35-49. 


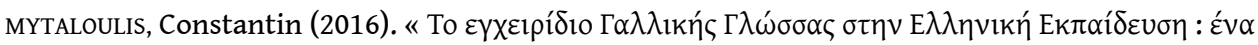

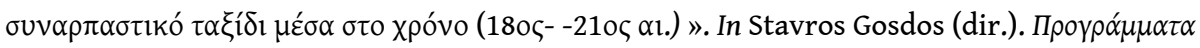

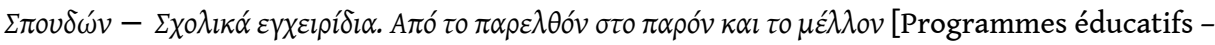
Manuels scolaires. Du passé au présent et à l'avenir]. Athènes : Collège Américain, 605-619. PROVATA, Despina \& VIHOU, Marina (2018). « La dimension culturelle dans les manuels illustrés pour enfants : le cas de La collection enfantine de Théodore Kyrios ». Documents pour l'histoire du français langue étrangère ou seconde, 60-61:323-343.

PUREN, Christian (2012 éd. numérisée [1988]). Histoire des méthodologies de l'enseignement des langues. (c) Christian PUREN.

REINFRIED, Marcus (1999). « Le mouvement réformiste et la méthode directe en Allemagne : développement, fondement théorique, variations méthodologiques ». Documents pour l'histoire du français langue étrangère ou seconde, 23 : 204-226. En ligne : [http://journals.openedition.org/ dhfles/3045].

VIGNER, Gérard (2017). Systématisation et maîtrise de la langue : l'exercice en FLE. Paris : Hachette.

\section{NOTES}

1. En ligne : [http://e-library.iep.edu.gr/iep/index.html].

2. L'école hellénique' comprenait les trois premières années du secondaire, et le 'gymnase', les quatre suivantes.

3. C'était un monopole sur la production et la diffusion des manuels. Des concours étaient lancés tous les quatre ans pour faire le choix des manuels officiels ( $c f$. Provata \& Vihou 2018 : 325).

4. En 1929, Socratis Sarivaxevanis déclare avoir tenu compte de toutes les nouvelles pratiques dans son manuel Méthode intuitive. Sur la méthode intuitive, une composante essentielle de la MD, cf Puren 2012 : 93-101.

5. Sur le développement de la méthodologie dite 'active' en France, $c f$ Puren 2012 : 142-162.

6. Sur ce problème, $c f$. Puren $2012: 168-169$.

7. C'est cette raison majeure - le fait que Dimitracopoulos ait voulu appliquer la MD intégrale jusqu'aux niveaux les plus avancés - qui a déterminé le choix de son Cours complémentaire pour la présente étude. L'ouvrage, en ce sens, est unique. De plus si on se fonde sur les critiques et opinions (très positives) concernant ses manuels, qui sont reproduites par l'auteur à la fin de ceux-ci et sont tirées de lettres de collègues, de pédagogues grecs et étrangers et de personnalités du monde de l'enseignement, on voit que la méthode de Dimitracopoulos a été grandement appréciée et utilisée et est donc représentative d'une époque importante dans la didactique du FLE en Grèce.

8. Voir Kakoyianni-Doa \& Monville-Burston (2019) pour plus de détails.

9. Même si les méthodologues de la MD n'étaient ni des théoriciens de linguistique générale ni des philosophes ( $c f$. Bouquet 1999 sur Wittgenstein et la MD), il est intéressant de faire des rapprochements, dans la perspective d'une histoire des idées, et de considérer que des transferts ont pu se faire entre savoirs théoriques et pratiques didactiques.

10. Cf. Puren (2012 [1988] : 78-79), sur l'intérêt porté aux aspects psychologiques de l'acquisition dans la MD.

11. Cf. le rapprochement que Bouquet (1999) fait entre le contenu des conversations dans la MD et les « jeux de langage » de Wittgenstein.

12. Les exercices sont absents dans les dernières leçons du livre. Peut-être est-ce pour encourager la lecture cursive à faire en dehors de la classe (cf. Puren 2012 : 113). 


\section{RÉSUMÉS}

L'article concerne le développement et la mise en œuvre de la méthodologie directe (MD) en Grèce dans l'enseignement $d u$ français. La première partie est consacrée au contenu des instructions ministérielles (1902-1961) qui préconisent l'utilisation de la MD dans le secondaire. L'analyse de ces documents permet d'établir quels aspects de la MD sont recommandés et l'évolution des instructions officielles au fil des ans, avec un regard particulier porté aux niveaux avancés. La seconde partie est une analyse du dernier manuel de la série Le français par la lecture et la conversation de Georges Dimitracopoulos. Il est montré que cet ouvrage se conforme fidèlement aux principes de la MD, bien que l'application intégrale de cette méthodologie soit moins aisée aux niveaux avancés.

The article concerns the reform movement as it developed in Greece for the teaching of French, as well as the implementation of the direct methodology (DM) in this country. The first part of the article is devoted to the contents of ministerial instructions (1901-1961) which prescribe the use of the DM in secondary schools. These documents are analysed to establish which aspects of DM are recommended and to describe the evolution of these instructions over the years. Particular attention is paid to advanced levels. The second part of the article is an analysis of the last textbook in the Français par la lecture et la conversation series by Georges Dimitracopoulos. It is shown that it wholly complies with the principles of DM, though the full application of this methodology is less easy at advanced levels.

\section{INDEX}

Mots-clés : enseignement du FLE, méthodologie directe (MD), Grèce, histoire des réglementations didactiques, MD aux niveaux avancés, cours avancé de Dimitracopoulos, méthodologie mixte

Keywords : teaching of French as a foreign language, direct method (DM), Greece, history of educational regulations, DM at advanced levels, Dimitracopoulos' advanced textbook, mixed methodology

\section{AUTEURS}

\section{FRYNI KAKOYIANNI-DOA}

Université de Chypre - frynidoa@ucy.ac.cy

\section{MONIQUE MONVILLE-BURSTON}

Université technologique de Chypre - burston.monique@cut.ac.cy 